\title{
Twisted mass fermions: neutral pion masses from disconnected contributions
}

\author{
F. Farchioni ${ }^{a}$, K. Jansen ${ }^{b}$, C. McNeile ${ }^{c}$, C. Michael ${ }^{c}$, I. Montvay ${ }^{d}$, K. Nagai ${ }^{b}$, M.
} Papinutto $^{b}$, J. Pickavance ${ }^{*}$, E. Scholz ${ }^{d}$, L. Scorzato ${ }^{e}$, A. Shindler ${ }^{b}$, N. Ukita ${ }^{d}$, C. Urbach $^{b f}$, U. Wenger ${ }^{b}$ and I. Wetzorke ${ }^{b}$

${ }^{a}$ Institut für Theoretische Physik, Universität Münster, Wilhelm-Klemm-Str. 9, 48149 Münster, Germany

${ }^{b}$ John von Neumann-Institut für Computing NIC, Platanenallee 6, 15738 Zeuthen, Germany

${ }^{c}$ Theoretical Physics Division, Dept of Mathematical Sciences, University of Liverpool, Liverpool L69 3BX, UK

${ }^{d}$ Deutsches Elektronen-Synchrotron DESY, Notkestr. 85, 22603 Hamburg, Germany

${ }^{e}$ Institut für Physik, Humboldt Universität zu Berlin, Newtonstr. 15, 12489 Berlin, Germany

${ }^{f}$ Institut für Theoretische Physik, Freie Universität Berlin, Arnimallee 14, 14195 Berlin, Germany

*jennypdiv.ac.uk

Twisted mass fermions allow light quarks to be explored but with the consequence that there are mass splittings, such as between the neutral and charged pion. Using a direct calculation of the connected neutral pion correlator and stochastic methods to evaluate the disconnected correlations, we determine the neutral pion mass. We explore the dependence on lattice spacing and quark mass in quenched QCD. For dynamical QCD, we determine the sign of the splitting which is linked, via chiral PT, to the nature of the phase transition at small quark mass.

XXIII International Symposium on Lattice Field Theory

25th-30th July 2005

School of Mathematics, Trinity College, Dublin

\footnotetext{
${ }^{*}$ Speaker.
} 


\section{TMQCD - Introduction}

TMQCD has Wilson quarks with the mass term modified by $m \rightarrow m+i \mu \tau_{3} \gamma_{5}$. In the continuum limit, a chiral rotation shows that this is equivalent to conventional QCD. At non-zero lattice spacing, however, the twisted mass formulation has several advantages, especially at maximal twist (where the effective mass term is imaginary). These advantages include (see [1] for a review)

- improved behaviour of inverters for small pion mass,

- order $a^{2}$ discretisation errors of flavour-conserving quantities, but with, however, the disadvantage that there will be flavour breaking effects (eg. $m\left(\pi^{+}\right) \neq m\left(\pi^{0}\right)$ ) at non-zero lattice spacing

Here we explore the $\pi^{+}, \pi^{0}$ mass difference both in quenched QCD to check scaling (with a) and in full QCD $\left(N_{f}=2\right)$ to explore the relationship via Chiral PT models to the nature of the phase transition at small quark masses.

At maximal $\left(90^{\circ}\right)$ twist, the operators appropriate to create hadrons are shown below:

hadron operator Disconnected piece

$\pi^{+} \quad \bar{u} \gamma_{5} d$

$\pi^{0} \quad \bar{u} I u+\bar{d} I d \quad \operatorname{Re} \operatorname{Tr} G_{u}$

$\eta^{0} \quad \bar{u} I u-\bar{d} I d \quad i \operatorname{Im} \operatorname{Tr} G_{u}$

$a_{0}^{+} \quad \bar{u} I d$

$a_{0}^{0} \quad \bar{u} \gamma_{5} u+\bar{d} \gamma_{5} d \quad \operatorname{Re} \operatorname{Tr} \gamma_{5} G_{u}$

$f_{0} \quad \bar{u} \gamma_{5} u-\bar{d} \gamma_{5} d \quad i \operatorname{Im} \operatorname{Tr} \gamma_{5} G_{u}$

where we have made use of the identity for propagators that $G_{d}=\gamma_{5} G_{u}^{\dagger} \gamma_{5}$. In several cases we need disconnected contributions: the notation is that $\operatorname{Tr} G_{u}(t)=\sum_{x} \bar{u}(x, t) u(x, t)$ and the disconnected contribution is the correlation of $G_{u}(0) G_{u}(t)$, with the vacuum piece of $G_{u}$ subtracted. This is illustrated by the right hand contribution below:

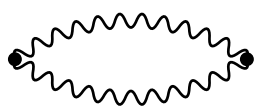<smiles>C1[As][As][13CH2][AsH2]1</smiles>

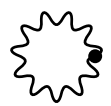

\section{Lattice evaluation of $\pi^{0}$ mass}

To evaluate the disconnected contributions to the hadronic correlation, we use a stochastic volume source with enough samples (24) that the stochastic sampling error is not dominant. We measure all $\bar{q} \Gamma q$ loops at all $\mathrm{t}$ (for zero momentum) and use both local and fuzzed sources. We use variance reduction [2] (gaussian random noise with subtraction of the lowest-order hopping parameter term) and we check the stochastic method by also evaluating the connected $\pi^{0}$ correlator which we compare with conventional (fixed source) evaluation.

For quenched lattices at $\beta=6.0$ and 5.85 with Wilson gauge action, $\kappa$ was chosen [3] to minimise parity mixing at $\mu=0$. The quenched results presented here are also reported in ref [ß].

As shown in fig. $1 \mathrm{a}$, we find that the disconnected contribution is dominant in the $\pi^{0}$ correlator. This is advantageous since the large signal is more easily evaluated using stochastic methods. 

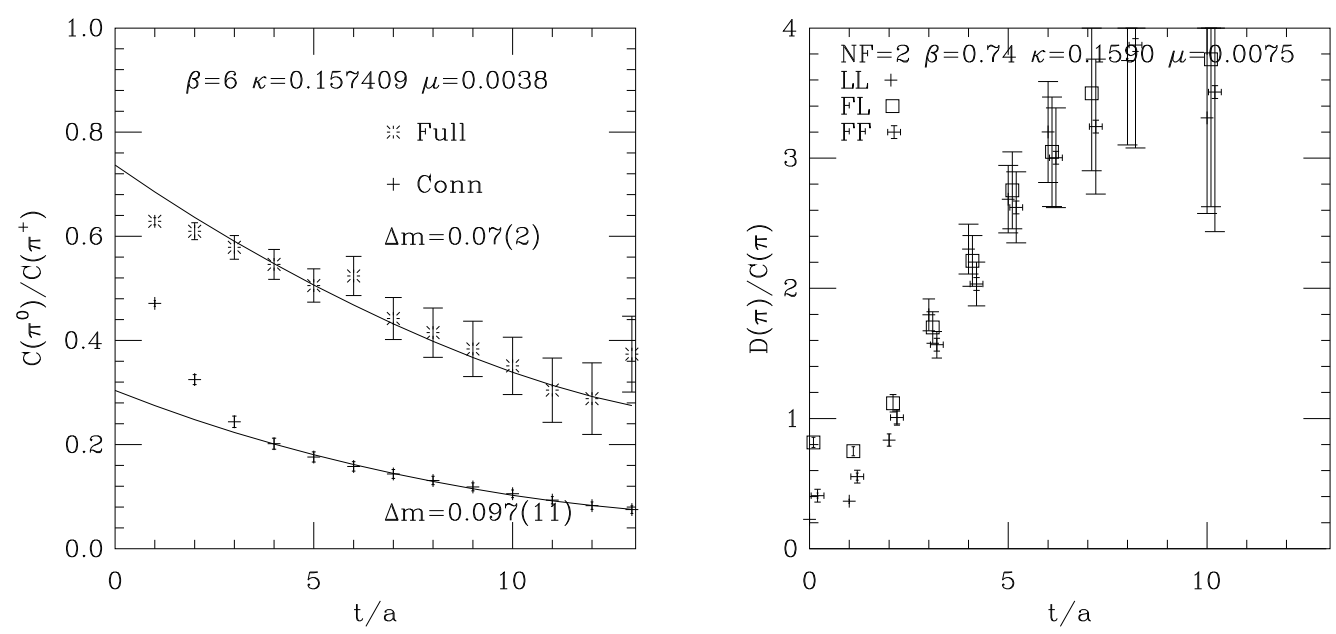

Figure 1: (a) Ratio of pion correlators (with local operators) for quenched fermions; (b) Ratio of disconnected to connected contribution to $\pi^{0}$ for dynamical fermions

Table 1: Pion masses (in lattice units)

\begin{tabular}{llllllllll}
\hline $\mathrm{N}_{f}$ & $\mathrm{~N}_{g}$ & $\beta$ & $\kappa$ & $\mathrm{a} \mu$ & $r_{0} / a$ & $\pi^{+}$ & $\pi^{0}$ (conn) & $\pi^{0}$ (tot) & $\pi^{0}-\pi^{+}$ \\
\hline 0 & 400 & 6.0 & .157409 & .0038 & 5.368 & $.1217(66)$ & $.218(9)$ & $.19(2)$ & $.07(2)$ \\
0 & 400 & 6.0 & .157409 & .0076 & 5.368 & $.1708(50)$ & $.246(5)$ & $.19(2)$ & $.02(2)$ \\
0 & 400 & 6.0 & .157409 & .0109 & 5.368 & $.2047(27)$ & $.267(4)$ & $.23(2)$ & $.02(2)$ \\
0 & 100 & 5.85 & .162379 & .0050 & 4.067 & $.1640(23)$ & $.319(14)$ & $.28(5)$ & $.12(5)$ \\
0 & 100 & 5.85 & .162379 & .0100 & 4.067 & $.2289(17)$ & $.351(8)$ & $.25(5)$ & $.02(5)$ \\
0 & 100 & 5.85 & .162379 & .0144 & 4.067 & $.2736(14)$ & $.380(6)$ & $.28(6)$ & $.00(6)$ \\
\hline 2 & 250 & .74 & .1590 & .0075 & 3.87 & $.1954(22)$ & $.37(4)$ & $.24(3)$ & $.04(3)$ \\
\hline 2 & 225 & 3.9 & .1610 & .0075 & 5.28 & $.214(7)$ & $.35(2)$ & $.27(2)$ & $.06(2)$ \\
\hline
\end{tabular}

We also plot the ratio of the full $\pi^{0}$ correlator to the $\pi^{+}$correlator, and this is not consistent with 1.0. We quantify this flavour splitting by fitting both $\pi^{0}$ and $\pi^{+}$correlators separately (using both local and non-local sources) and we show our results in Table 1.

As well as evaluating the full $\pi_{0}$ correlator, we can take advantage of the more accurate results available for the connected contribution to the $\pi^{0}$ by interpreting [3] this contribution as from a (quenched) theory with fermion action with mass term $m+i \mu \gamma_{5}$. That theory has no disconnected contribution to $\pi_{0}$ (since isospin is now a good symmetry) but the difference between it and the TMQCD value for the $\pi^{+}$mass can be used to explore lattice artifacts. These results are illustrated in fig. 2. Here we see that the $\pi^{0}-\pi^{+}$mass difference decreases consistently with $a^{2}$ as $a \rightarrow 0$. Moreover, we find that we can summarise the splitting by $r_{0}^{2}\left(m^{2}\left(\pi^{0}\right)-m^{2}\left(\pi^{+}\right)\right) \approx c\left(a / r_{0}\right)^{2}$, with the difference smaller including the disconnected contribution to the $\pi^{0}(c \approx 10$ but with large error) than connected alone ( $c \approx 23$ and quite well determined).

We also explore the $\pi^{0}$ mass with dynamical fermions [4] - using $N_{f}=2$ flavours of quarks ( $u$ and $d$ ). We fitted the $2 \times 2$ matrix of local and fuzzed correlators where both connected and disconnected contributions were here evaluated stochastically. We concentrate on results with ap- 

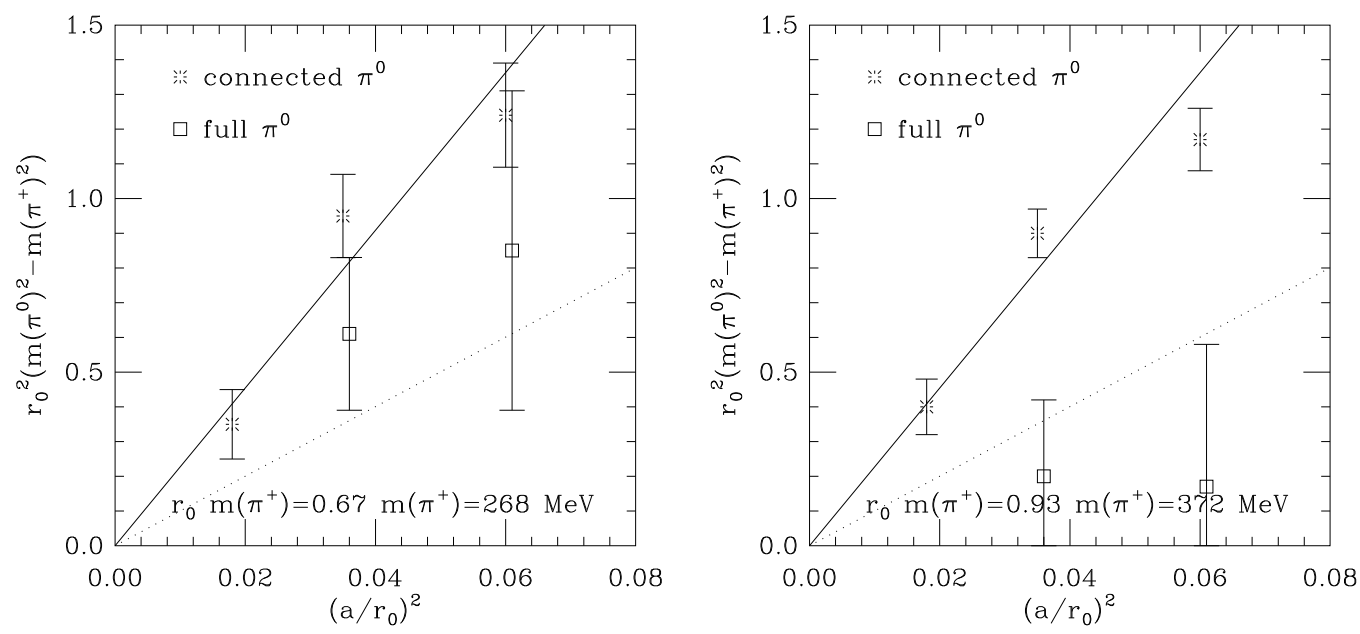

Figure 2: Mass splittings between $\pi^{0}$ and $\pi^{+}$in quenched studies.

proximately maximal twist. Using the DBW2 gauge action $(\beta=0.74)$ (see also fig. 1b), the pion masses are recorded in Table 1. The mass splitting $m\left(\pi^{0}\right)-m\left(\pi^{+}\right)$is consistent with zero but with a sign which corresponds (via leading order $\chi$ PT [ []]) to an Aoki phase at small quark mass. We also analyse results from the tree-level improved Symanzik gauge action (TLS $\beta=3.9$ ) which are also presented in Table 1 . The case nearest to maximal twist again shows a mass splitting $m\left(\pi^{0}\right)-m\left(\pi^{+}\right)$with a sign which corresponds with similar assumptions to an Aoki phase.

\section{Other mesons}

The flavour singlet pseudoscalar ( $\eta$ for $N_{f}=2$ ) involves a disconnected contribution even for conventional Wilson fermions. Here we evaluate the contribution for our twisted data sets. The full contribution is $C-D$ where $C$ is connected and $D$ is disconnected. Positivity then requires $C-D>0$ or, equivalently, $D / C<1$.

We see, in fig. 3a that this requirement is violated in quenched QCD. Quenched QCD has ghost states and the flavour-singlet sector is not physical. For $N_{f}=2$, however, the requirement $D / C<1$ is well satisfied and it would be possible to extract the $\eta$ mass from the rate at which it approaches 1 , albeit with large errors. This is confirmation that the algorithms used to evaluate dynamical gauge configurations are consistent with positivity requirements.

We also explore the spectrum of scalar mesons. In non-twisted QCD, the flavour non-singlet $a_{0}$ receives only connected contributions while the singlet $f_{0}$ receives both connected and disconnected. In TMQCD, the $a_{0}$ states are again split with the $a_{0}^{+}$having only connected contributions whereas $a_{0}^{0}$ has both. As shown in fig. 4, the disconnected contribution to $a_{0}^{0}$ does help to make it closer to the charged $a_{0}$, although there is a residual flavour violation (that we find decreases as the lattice spacing decreases).

Again, because this time of the decay to $\eta \pi$, the quenched correlators are unphysical (they are negative). The $N_{f}=2$ case, however, shows that positivity is restored, as it should be. 

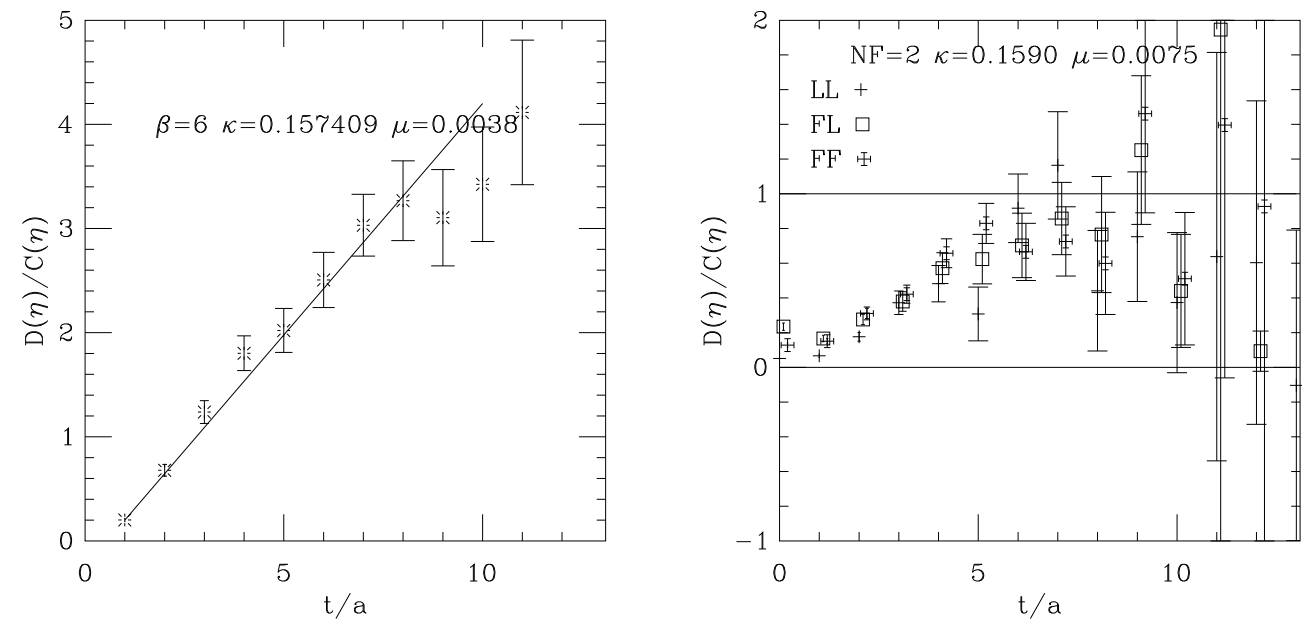

Figure 3: Flavour singlet pseudoscalar meson: ratio of disconnected to connected contribution to correlations. Here the total is $C-D$, so positivity implies $D / C<1$. (a) quenched and (b) with $N_{f}=2$.

In detail, the scalar meson contributions will have to be corrected for the small admixture of pseudoscalar meson arising if the mixing angle is not exactly $90^{\circ}$. This small admixture becomes significant at larger $t$ because the pseudoscalar mass is lighter than the scalar mass.
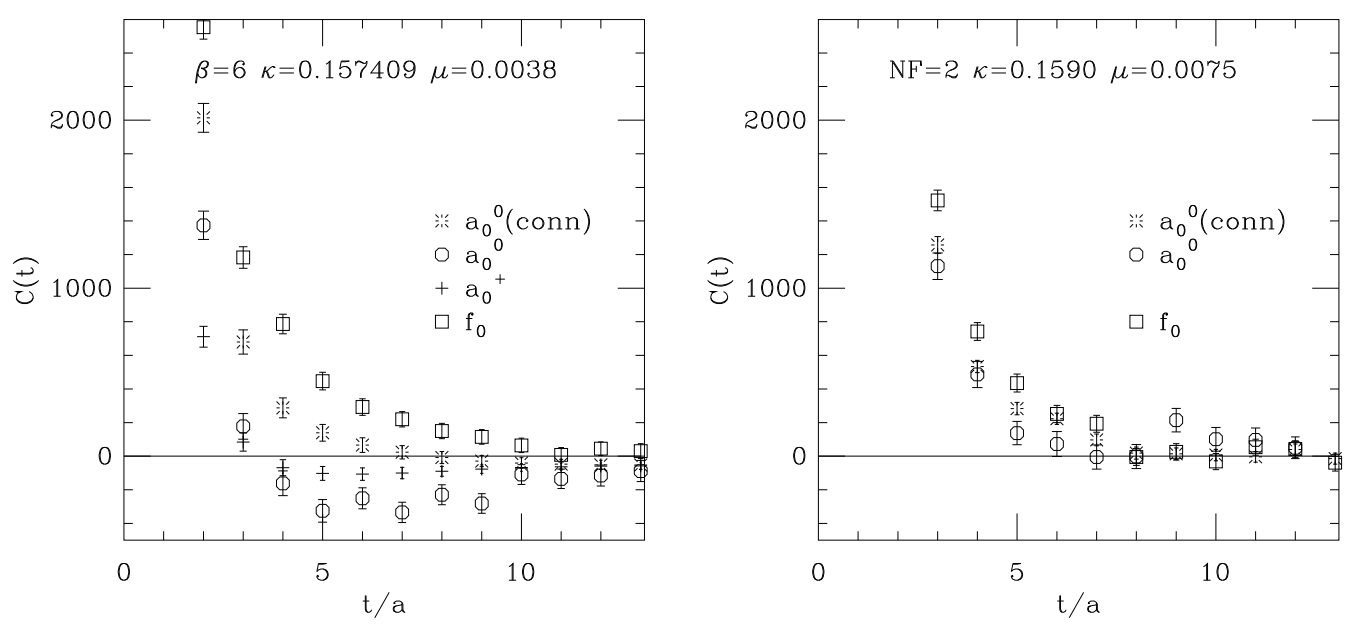

Figure 4: Scalar meson contributions (a) quenched and (b) with $N_{f}=2$

\section{Summary}

- The disconnected contribution to $\pi^{0}$ is big compared to the connected.

- The $\pi^{+} \pi^{0}$ mass difference decreases with decreasing $a$ as $r_{0}^{2}\left(m^{2}\left(\pi^{0}\right)-m^{2}\left(\pi^{+}\right)\right) \approx c\left(a / r_{0}\right)^{2}$ in a quenched study with $c \approx 10$.

- From a full-QCD study, one can extract the chiral lagrangian parameter relevant to $\pi^{0} \pi^{+}$ splitting. For the DBW2 gauge action, the splitting is small (consistent with zero within 
errors) with a sign which corresponds to an Aoki phase at small quark mass. For the TLS gauge action, the same sign is also seen.

- The flavour singlet pseudoscalar meson signal $(\eta)$ is sensible and useful for $N_{f}=2$ with dynamical TMQCD.

- For scalar mesons, the need for disconnected diagrams and the possibility of the signal being polluted by mixing (if twist not $90^{\circ}$ ) with pseudoscalar suggests this will be a difficult area.

\section{References}

[1] A. Shindler, Twisted mass lattice QCD: Recent developments and results, PoS(LAT2005)014.

[2] UKQCD Collaboration, C. McNeile and C. Michael, Mixing of scalar glueballs and flavour-singlet scalar mesons, Phys. Rev. D63 (2001) 114503, hep-lat/0010019.

[3] XLF Collaboration, K. Jansen, C. McNeile, C. Michael, K. Nagai, M. Papinutto, J. Pickavance, A. Shindler, C. Urbach, and I. Wetzorke, Flavour breaking effects of Wilson twisted mass fermions, hep-lat/0507032.

[4] F. Farchioni et al., PoS(LAT2005)072.

[5] S. R. Sharpe and J. M. S. Wu, Twisted mass chiral perturbation theory at next-to-leading order, Phys. Rev. D71 (2005) 074501, hep-lat/0411021]. 